\title{
Reducing Traffic Congestion in Urban Areas via Real-Time Re-Routing: A Simulation Study
}

\author{
Mauro Vallati ${ }^{10000-0002-8429-3570]}$ and Lukáš Chrpa ${ }^{20000-0001-9713-7748]}$ \\ 1 University of Huddersfield, Huddersfield, United Kingdom \\ 2 Artificial Intelligence Center, Czech Technical University in Prague, \\ Czech Republic
}

\begin{abstract}
Traffic congestion problems of urban road networks are having a strong impact on economy, due to losses from accidents and delays, and to public health. The recent progress in connected vehicles is expanding the approaches that can be exploited to tackle traffic congestion, particularly in urban regions. Connected vehicles pave the way to centralised real-time re-routing, where a urban traffic controller can suggest alternative routes to be followed in order to reduce delays and mitigate congestion issues in the network. In this work, we introduce a centralised architecture and we compare in simulation a number of approaches that can be exploited for re-routing vehicles.
\end{abstract}

Keywords: Urban Traffic Control · Real-time Re-Routing · Traffic Optimisation

\section{Introduction}

Nowadays, the traffic in urban areas becomes one of the major economical problems due to losses from traffic accidents and travel delays. In rush hours, the problem with traffic jams, road congestion and travel delays is far more apparent. With continuing growth of global urbanisation, the problem with traffic congestion is expected to exacerbate. Hence there is a need for intelligent traffic control techniques that can, at least to some extent, mitigate the problem. With the recent progress in the area of connected vehicles we have more options for designing intelligent traffic control techniques [8].

One of the well studied options is to control traffic lights in order to optimise traffic flow in intersections. Such techniques include SCOOT [1], MOVA [10] or SCATS [5] that adapt green phase lengths according to current traffic conditions. Apart of reactive techniques, there are techniques leveraging Automated Planning that take into account longer time period in which traffic in the urban road network evolves $[9,7]$.

Intelligent traffic light control, however, is only able to tackle one aspect of urban traffic congestion as it can only affect bandwidth of intersections. A complementary approach that can be exploited to mitigate road congestion is intelligent vehicle routing. That is, in a nutshell, routing vehicles from their 
locations of origin to their destination location throughout the road network in a smart way -maximising some aspects specified by the traffic controller. In consequence, two different vehicles might take different routes even though they have identical locations of origin and destination. Leveraging Automated Planning to tackle the problem of intelligent vehicle routing throughout the road network achieved promising results $[3,2]$. Those techniques approach the problem from centralised perspective, i.e., there is a central urban traffic controller that suggests routes to all the navigating vehicles [11].

One of the known drawbacks of centralised approaches is the scalability issue: since a controller is in charge of a whole region, it may struggle in the presence of a large number of vehicles. Overcoming the scalability issue can be done, for example, by considering computationally inexpensive metrics for re-routing vehicles - so that the central controller can more easily deal with large volumes of traffic. Another opportunity is to consider decentralised approaches, where each vehicle can decide in isolation the best route to select. While this is the most common approach currently exploited, as typical satellite navigators are based on this decentralised perspective, it can be suboptimal since it does not take into account the behaviour of the other vehicles, and the likely evolution of the network conditions. For the interested reader, a comparison between centralised and decentralised approaches for traffic light controlling has been recently performed by Manolis et al. [6].

In this paper, we address the problem of intelligent vehicle rerouting in realtime. We introduce an architecture that supports the real-time re-routing of vehicles approaching a controlled urban region, and we exploit such architecture to compare a number of criteria that can be used to re-route vehicles with the aim of reducing the congestion of the network links. We specified six different criteria that determine whether the vehicle should re-route. Thee of the criteria consider the current situation in the road network, and can therefore be exploited also in a decentralised fashion where each vehicle is considering the condition in isolation, while the other three criteria consider estimates of the near future traffic situation, and are typical of a centralised approach where a traffic controller can predict the evolution of the network conditions. Effectiveness of the criteria is evaluated on the Milton Keynes road network while considering historical data from the morning rush hour traffic. The results indicate that criteria based on the current or (near) future road occupancy are promising for effective and efficient vehicle re-routing, when a large number of vehicles are connected and are following the provided suggestions.

\section{Investigated Architecture and Approaches}

This section is devoted to describe the investigated architecture, and the metrics considered to be used for performing the real-time re-routing of vehicles, in order to spread vehicles in the urban network with the aim of reducing congestion.

Figure 1 provides an overview of the architecture that is designed and considered for this investigation. The main aim of the framework is to support the exploitation of techniques for maximising the use of the controlled urban 


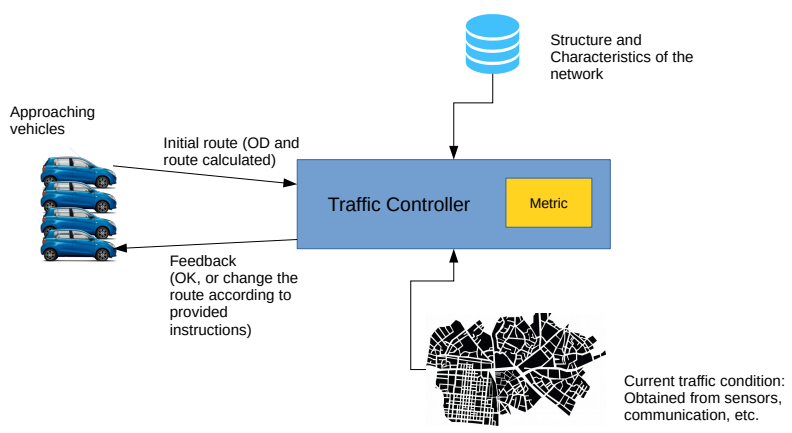

Fig. 1. An overview of the considered architecture.

network via real-time re-routing, each vehicle approaching the controlled area will receive re-routing instructions based on its current destination, and on the current traffic conditions.

The main element of the framework is the traffic controller; by taking into account the vast amount of available information and data, the controller is in charge of deciding what are the best routes to be followed by the connected vehicles that are approaching the network, according to the considered optimisation metric. The traffic controller relies on three types of input:

- Structure and characteristics of the network; This includes a description of the topology of the network in terms of links, junctions, traffic lights, etc.

- Current traffic condition; Available sensors distributed in the controlled region can provide additional information about the status, for instance in terms of air quality, congestion, accidents, etc. Further, connected vehicles can directly communicate with the traffic controller, providing information about their current location, speed, etc.

- Approaching vehicles; Connected vehicles that are approaching the controlled urban area can communicated with the controller in order to share their current path, and their destination.

On the basis of the available information, and taking into account the metric to optimise, the traffic controller is in the best position for deciding the route to be assigned to the approaching vehicles. They can of course maintain their current route, or they can be assigned a different one. For the sake of this analysis,and following the common practice, here we consider a path (or a route) to be an ordered sequence of links that a vehicle has to navigate for reaching its destination.

We designed the traffic controller to provide re-routing instructions to vehicles entering the controlled region. For every approaching vehicle, or every $x$ seconds, the controller assess the condition of the network and, considering the metric to be optimised, takes a decision. In other words, the controller does not built a single overall simulation of the area to control, but keeps re-assessing 
conditions. The decision to allow re-routing only for approaching vehicles has been taken for two main reasons: (i) to reduce the complexity on the controller side, and (ii) to avoid numerous re-routing for a vehicle. In particular, the latter point is of primary importance in cases where the vehicle is connected, but not autonomous, and therefore a human driver has to accept re-routing instructions and modify the path accordingly.

\section{$2.1 \quad$ Metrics}

A key element of the investigated architecture, that is a blue print for a connected urban traffic controller, is of course the metric to be used to re-route approaching vehicles, with the aim of optimising the traffic conditions of the network.

Our analysis is focused on metrics that can be computed in real-time, and are therefore computationally cheap, and that can allow to compare two or more paths. The aim is to be able to decide the path that is expected to be less congested, in order to distribute vehicles.

We identify two main classes of metrics that can be designed: metrics that gives an overview of the current network conditions, and metrics that aim at predicting the evolution of traffic conditions. For the sake of operationality, i.e. making sure that the re-routing can be done in real-time, the prediction of the evolution of the traffic conditions has to leverage on features and elements that can be easily computed, also in large urban areas. For the first class of approaches, we designed the following metrics:

- Occupancy. the current occupancy of a path $P$ is calculated as the total number of vehicles across all the links that are part of the path $P$. Formally:

$$
\operatorname{occ}(P)=\sum_{n \in P} \text { vehicles }(n)
$$

Where vehicles $(n)$ represents the number of vehicles that are currently on link $n$, either moving or queuing.

- Density. The proposed measure of occupancy does not take into account the macimum capacity of a link, in terms of the maximum number of vehicles that can physically fit it. This may potentially lead to cases where paths composed by short extremely congested links are preferred over paths that include longer links. To address this potential issue, the notion of Density takes also into account the maximum capacity of each link, in order to provide a more accurate overall evaluation of the congestion:

$$
\operatorname{density}(P)=\sum_{n \in P} \frac{\operatorname{vehicles}(n)}{\text { maxCapacity }(n)}
$$

For the sake of readability, we assume that the density function can also accept a single link as an input parameter. In that case, the density of the single provided link is returned. 
- Journey time. The current estimation of the journey time for a path $P$ is calculated as a sum of travel times for each link (note that waiting time at intersections is incorporated in the link travel time):

$$
\operatorname{travel} T(P)=\sum_{n \in P} \operatorname{travel} T(n)
$$

Where travelT $(n)$ represents the most recent travel time measurement for link $n$. If no measure is available, then the minimum "physical" travel time is considered, as the ratio between the length of the link and the allowed maximum speed. Notably, this is the metric traditionally leveraged by connected Satellite Navigators such as Waze ${ }^{3}$ and Navfree ${ }^{4}$, as is therefore deemed to be extremely informative.

The second class of approaches focuses on providing an estimation of the evolution of the traffic conditions on the considered paths. We will later refer to this class as "cumulative", and it includes the following metrics:

- Cumulative occupancy. The cumulative occupancy of a path $P$ is calculated as a sum of the number of vehicles that are currently passing the links or are routed through them in future:

$$
\operatorname{cumulativeOcc}(P)=\sum_{n \in P} \sum_{v \in V} \operatorname{expected}(v, n)
$$

Where $\operatorname{expected}(v, n)$ represents the expected number of vehicles to navigate through link $n$, and is calculated as follows.

$$
\operatorname{expected}(v, n)=\left\{\begin{array}{l}
1: n \in \operatorname{path}(v) \\
0: \text { otherwise }
\end{array}\right.
$$

$\operatorname{path}(v)$ represents the remaining path that vehicle $v$ has yet to navigate in order to reach its destination. In other words, the cumulative occupancy value of a path gives a very pessimistic overview of traffic conditions, by assuming that all the vehicles in the network, that are expected to navigate through some links of the path, are occupying all the links at the same time. It is as if a vehicle is actually "booking" all the links that it is planned to navigate in the future. This gives an unrealistic view of the traffic conditions to the traffic controller, but forces the controller to pre-actively take action in order to distribute traffic as much as possible from early stages.

- Cumulative density. This is an extension of the notion of density, where the cumulative occupancy of each link is considered, instead of the current occupancy.

$$
\operatorname{cumulDensity}(P)=\sum_{n \in P} \frac{\sum_{v \in V} \operatorname{expected}(v, n)}{\max \operatorname{Capacity}(n)}
$$

\footnotetext{
${ }^{3}$ https://www.waze.com/

${ }^{4}$ http://navfree.android.informer.com/
} 
As for the cumulative occupancy, also the cumulative density gives a pessimistic overview of the traffic conditions, but it is aiming at providing an estimation of how much links may become congested, rather than providing the raw number of expected vehicles.

- Cumulative journey time. This measure is a combination of the previously described cumulative occupancy and journey time metrics. The idea is to acknowledge the fact that an increased occupancy is likely to have a detrimental impact on the travel time of a path. Therefore, the estimate of the travel time of a path is increased according to the calculated cumulative occupancy, as follows:

$$
\text { cumulTravelT }(P)=\sum_{n \in P} \frac{\operatorname{travel} T(n) \times \sum_{v \in V} \operatorname{expected}(v, n)}{\text { vehicles }(n)}
$$

In cases where vehicles $(n)=0$, only travelT $(n) \times \operatorname{density}(n)$ is considered for a link $n$. The above equation has been designed so that future travel time is directly affected by the cumulative occupancy. Since the cumulative occupancy is generally expected to be higher than the maximum link capacity, due to the way in which it is calculated, this measure is ignoring that travel time is not a linear function of the number of vehicles. This has also been done to reduce the complexity of calculating the metric, and to ensure that it can cope also with unexpected travel conditions.

It is worth noting that the cumulative dimension of the metrics can be optimised according to the characteristics of the network, and of the traffic. In the above definitions we considered that a vehicle is occupying at the same time, all the links of its current path. However, this can be modified in order to consider only the next few links, or the links corresponding to the next 1 kilometre of the path.

\section{Simulation Investigations}

\subsection{Setup}

To study and compare the performance of the six introduced metrics for real-time re-routing of connected vehicles in urban areas under realistic traffic conditions, the network of Milton Keynes centre has been used. In particular, here we consider a SUMO microsimulation model [4], and the network is shown in Figure 2. Milton Keynes is a town of the United Kingdom, located about 80 kilometres north-west of London. Milton Keynes has a population of approximately 230,000 . The model covers an area of approximately 2.9 square kilometres, and includes more than 25 junctions and more than 50 links.

The model simulates the morning rush hour, and has been built by considering historical traffic data collected between 8am and 9am on non-holiday weekdays. Data has been provided by the Milton Keynes Council, and gathered by sensors distributed in the region between December 2015 and December 2016 . 


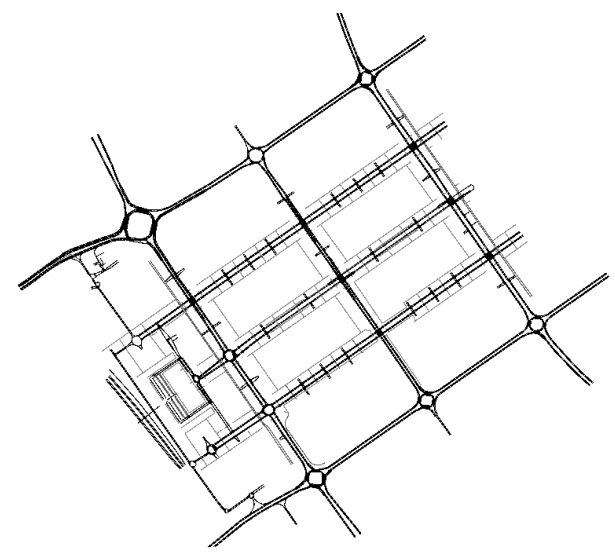

Fig. 2. The modelled central Milton Keynes urban area. Large influx of traffic, for the considered morning peak time, comes from the West roundabout, and from the North East roundabout.

Table 1. Performance of the cumulative metrics when run in simulation with normal traffic conditions, and all vehicles are following the re-routing instructions of the traffic controller. Occ, Den, and JourneyT stand for, respectively, Occupancy, Density, and Journey Time. A-* indicates that all the remaining path is considered as occupied by a vehicle. $5^{-*}$ is used to indicate that at most the next 5 links are considered occupied. Bold is used to highlight best performance.

\begin{tabular}{l|c|c|c|c|c|c} 
& \multicolumn{5}{|c}{ Considered Metrics } \\
& A-Occ & A-Den & A-JourneyT & n5-Occ & n5-Den & n5-JourneyT \\
\hline \hline departed vehicles [\#] & $\mathbf{1 8 8 2}$ & 1731 & 1842 & 1860 & 1823 & 1848 \\
arrived vehicles [\#] & 1533 & 1045 & 1605 & $\mathbf{1 6 6 8}$ & 1443 & 1634 \\
avg, speed [m/s] & 2.8 & 0.9 & 3.9 & $\mathbf{4 . 8}$ & 2.5 & 4.0 \\
avg, trip length [m] & $\mathbf{1 9 3 7 . 0}$ & 1942.7 & 2079.7 & 2049.5 & 2055.4 & 2120.9 \\
avg, trip duration [s] & 476.7 & 696.1 & 404.7 & $\mathbf{3 5 6 . 5}$ & 532.1 & 383.3 \\
avg, time loss [s] & 335.7 & 555.0 & 253.1 & $\mathbf{2 0 7 . 2}$ & 382.6 & 228.8 \\
\hline
\end{tabular}

Traffic signal control information has been provided again by the Council. The model has been calibrated and validated.

The presented architecture has been implemented in Python, and uses the TRaCI interface to interact with the SUMO simulation environment, in order to get the current network status, communicate with approaching vehicles, and inform vehicles of re-routing. For every couple of origin-destination, described by the traffic flows of the model, between 2 and 3 alternative routes are considered for distributing traffic. Such routes have been provided by a human expert, that has a good understanding of the dynamics of the modelled region. Alternatives can, in principle, be automatically calculated (by using approaches based on the Dijkstra algorithm, for instance), relying on human expertise can allow to exploit some insights that are based on knowledge that is not captured by the symbolic model of the network. The simulation is run for 1 hour and then stopped. For 
Table 2. Performance of the considered metrics when run in simulation with normal traffic conditions, and all vehicles are following the re-routing instructions of the traffic controller. Default indicates that no traffic control is in use. Occ, Den, and JourneyT stand for, respectively, Occupancy, Density, and Journey Time metrics. C-* is used to indicate the cumulative version of the corresponding metric. Best performance are in bold.

\begin{tabular}{l|c|c|c|c|c|c|c} 
& & \multicolumn{6}{|c}{ Considered Metrics } \\
& Default & Occ & Den & JourneyT & C-Occ & C-Den & C-JourneyT \\
\hline \hline departed vehicles [\#] & 1669 & 1818 & 1808 & 1842 & $\mathbf{1 8 6 0}$ & 1823 & 1848 \\
arrived vehicles [\#] & 801 & 1628 & 1611 & 1572 & $\mathbf{1 6 6 8}$ & 1443 & 1634 \\
avg, speed [m/s] & 0.6 & 4.6 & $\mathbf{5 . 2}$ & 3.7 & 4.8 & 2.5 & 3.9 \\
avg, trip length [m] & 2297.4 & 2098.5 & 2136.6 & 2059.9 & $\mathbf{2 0 4 9 . 5}$ & 2055.4 & 2121.0 \\
avg, trip duration [s] & 788.9 & $\mathbf{3 5 4 . 3}$ & 378.2 & 407.7 & 356.5 & 532.1 & 383.3 \\
avg, time loss [s] & 622.1 & $\mathbf{2 0 1 . 6}$ & 222.7 & 257.5 & 207.2 & 382.6 & 228.8 \\
\hline
\end{tabular}

each set of experiments, the simulation is run five times and results are averaged, to account for non-determinism.

The simulation results are summarised in terms of the following SUMOcalculated performance indices:

- number of departed (arrived) vehicles. Indicates the number of vehicles that entered the region (reached destination) during the simulation. A vehicle can enter the region if the entry point link has enough space to accommodate it, otherwise it is assumed to be queuing outside the region.

- average speed $(\mathrm{m} / \mathrm{s})$ of the vehicles.

- average trip length $(\mathrm{m})$ and duration $(\mathrm{s})$. Length and duration reports the average measurement of the trips of the vehicles to reach their destination from the entry point.

- Average time loss (s). This value indicates the time that has been lost due to vehicles queuing, or travelling at a speed that was lower than the maximum allowed.

\subsection{Results and Discussions}

A first pivotal aspect to investigate, for metrics of the cumulative class, is to identify the most promising number of links to be considered as occupied by a vehicle that is following an assigned path. After a number of preliminary experiments, we found that a lookahead of 5 links allows the traffic controller exploiting the metrics to deliver consistently good performance. Table 1 shows the performance of the cumulative metrics when a vehicle is occupying (A)ll the remaining links of the path, or only the next 5 (n5). In most of the cases, the n5cumulative version allows the corresponding metric to deliver better performance than the All one. Given the presented results, in the remainder of this analysis we will use the $\mathrm{n} 5$ - version for the cumulative class of metrics.

Having identified the most promising value to be used by the cumulative metrics, we can now turn our attention to an extensive comparison of the considered techniques. In the first scenario, we consider ideal conditions: traffic is 


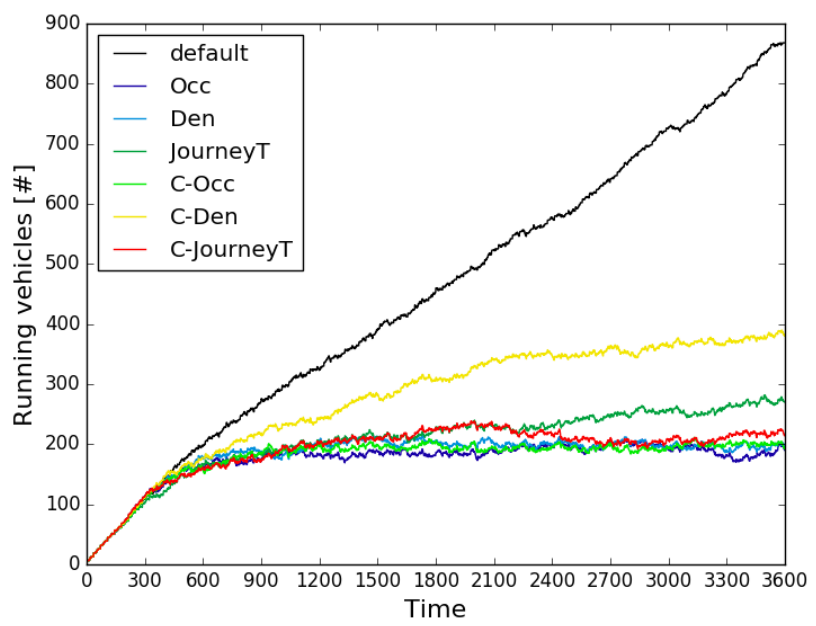

Fig. 3. The number of vehicles running, i.e. navigating the controlled region, at any point in time during the simulations. Occ, Den, and JourneyT stand for, respectively, Occupancy, Density, and Journey Time metrics. C-* is used to indicate the cumulative version of the corresponding metric.

normal -as for the historical data collected in the area- and all the vehicles are connected and are following the re-routing instructions provided by the traffic controller. This scenario should provide an idea of how different metrics can help to distribute traffic in the urban region when used under the best possible conditions; it is a sort of upper bound of the achievable performance. The results are presented in Table 2, and includes the default performance of the network, achieved when no traffic control is in operation. In the Default settings, vehicles enter the network and follow their pre-calculated path to the destination, that does not take into account the network conditions. As expected, all the techniques are significantly outperforming the default; this is an indication that the proposed architecture can effectively reduce traffic congestion via re-routing. Notably, there is no metric that allows to deliver the best performance according to all the indicators. In general, Occupancy and Cumulative Occupancy seem capable of delivering the best performance, and show very similar results. Metrics based on the notion of Density do not perform well when the cumulative version is used, as a limited number of vehicles can reach its destination during the simulation time. Remarkably, the use of Density allows to achieve the highest average speed of vehicles in the network, even though at the cost of longer journeys on average. With regards to the Journey time metrics, the use of the cumulative version allows to deliver consistently good performance.

In order to shed some light on the performance of the compared techniques, Figure 3 shows the evolution over time of the number of vehicles running in the controlled region. In other words, the number of vehicles that entered the 
Table 3. Performance of the considered metrics when run in simulation with heavy traffic conditions, and all vehicles are following the re-routing instructions of the traffic controller. Default indicates that no traffic control is in use. Occ, Den, and JourneyT stand for, respectively, Occupancy, Density, and Journey Time metrics. C-* is used to indicate the cumulative version of the corresponding metric. Best performance are in bold.

\begin{tabular}{l|c|c|c|c|c|c|c} 
& & \multicolumn{6}{|c}{ Considered Metrics } \\
& Default & Occ & Den & JourneyT & C-Occ & C-Den & C-JourneyT \\
\hline \hline departed vehicles [\#] & 1869 & $\mathbf{2 9 4 5}$ & 2778 & 2678 & 2919 & 2215 & 2858 \\
arrived vehicles [\#] & 495 & 2071 & 2054 & 1844 & $\mathbf{2 0 7 5}$ & 1425 & 2008 \\
avg, speed [m/s] & 0.2 & $\mathbf{1 . 9}$ & 1.7 & 1.2 & 1.6 & 1.1 & 1.6 \\
avg, trip length [m] & 2295.0 & 2117.4 & 2164.5 & 2108.7 & $\mathbf{2 0 9 7 . 4}$ & 2191.5 & 2159.0 \\
avg, trip duration [s] & 1107.5 & 777.6 & $\mathbf{6 5 9 . 3}$ & 714.4 & 722.4 & 891.5 & 704.7 \\
avg, time loss [s] & 935.0 & 623.5 & $\mathbf{5 0 2 . 0}$ & 561.1 & 569.6 & 732.0 & 547.6 \\
\hline
\end{tabular}

area but has not yet reached the destination. This indicator can help relating and understanding the departed and arrived vehicles measurements provided in Table 2. On the one hand, the number of arrived vehicles are an important factor that shows the ability of the technique in letting traffic move, while the departed vehicles indicator focuses on the ability of the traffic controller in maintaining the entry points of the network free. The number of running vehicles shows to us what happens between these 2 extremes. From this perspective, it is easy to notice that the Cumulative Density metrics is having issues in keeping the traffic moving in the region, with approx. 400 vehicles in the region at the end of the simulation time. The Journey time metric is facing some issues as well, but those are less pronounced. The remaining techniques are all showing similar performance with regards to this indicator, having approx. 200 vehicles in the region at the end of the simulation time.

We then considered a second scenario, with heavy traffic conditions. To simulate heavy traffic, we assumed that twice the normal amount of vehicles $(3,800)$ are accessing the network over the same 1 hour simulation time. We are again assuming that all the vehicles are connected and are following the re-routing instructions. Table 3 shows the results of the performed analysis. Under such circumstances, the improvement that can be obtained by using the investigated framework, compared to the default network behaviour, is even more significant. Under default conditions, 495 vehicles would reach their destination within the simulation time: the worst metric is allowing 1,425 vehicles to reach their destination, with an improvement of approx $290 \%$. The Density metric seems to exploit well the available network, by minimising time loss and the average trip duration. In terms of trips length and number of vehicles arrived / departed, the Occupancy-based metrics are still delivering the best performance. As before, the Journey time metrics do not seem to be capable of effectively re-route the traffic in the network.

Finally, our third scenario investigates the importance of penetration rate for the proposed metrics. Table 4 shows the performance achieved when the traffic 
Table 4. Performance of the considered metrics when run in simulation with normal traffic conditions, and $50 \%$ of the vehicles are following the re-routing instructions of the traffic controller. Default indicates that no traffic control is in use. Occ, Den, and JourneyT stand for, respectively, Occupancy, Density, and Journey Time metrics. C-* is used to indicate the cumulative version of the corresponding metric. Best performance are in bold.

\begin{tabular}{l|c|c|c|c|c|c|c} 
& & \multicolumn{5}{|c}{ Considered Metrics } \\
& Default & Occ & Den & JourneyT & C-Occ & C-Den & C-JourneyT \\
\hline \hline departed vehicles [\#] & 1669 & 1801 & 1787 & 1784 & $\mathbf{1 8 0 7}$ & 1789 & 1797 \\
arrived vehicles [\#] & 801 & $\mathbf{1 5 4 0}$ & 1484 & 1464 & 1539 & 1454 & 1465 \\
avg, speed [m/s] & 0.6 & $\mathbf{3 . 9}$ & 2.8 & 3.0 & 3.7 & 3.1 & 2.8 \\
avg, trip length [m] & 2297.4 & 2303.5 & 2330.1 & 2288.0 & 2280.0 & $\mathbf{2 2 6 4 . 3}$ & 2310.1 \\
avg, trip duration [s] & 788.9 & $\mathbf{4 3 4 . 0}$ & 466.8 & 493.3 & 439.1 & 499.8 & 491.1 \\
avg, time loss [s] & 622.1 & $\mathbf{2 6 6 . 6}$ & 297.4 & 327.0 & 273.3 & 335.4 & 323.24 \\
\hline
\end{tabular}

condition is normal, but only $50 \%$ of the vehicles are following the provided re-routing instructions. By considering the results presented in Table 2, it is apparent that the Density and Journey time metrics are the most sensitive to the penetration rate, as its performance are the most affected by the reduction of vehicles following instructions. On the contrary, Occupancy-based metrics are robust with regards to this aspect, and are still capable of delivering generally good performance according to the indicators.

\section{Conclusion}

In this paper, we performed an extensive simulation study aimed at comparing six criteria for real-time re-routing of connected vehicles in an urban region. Vehicles are re-routed in order to minimise congestion in some part of the network, by spreading the traffic. The criteria are divided into two main classes, one that considers only the current condition of the network for re-routing vehicles, and the other that is also taking into account some prediction of the traffic evolution. To ensure the exploitability of the criteria in a real-world scenario, we focused on aspects that are computationally cheap to assess, in order to support a centralised urban traffic control architecture.

The simulation study considered the central Milton Keynes urban area in the morning rush hour. Data has been provided by the Milton Keynes council, and the considered model has been calibrated and validated. The experimental results indicated that: (i) Journey time, that is the criteria most used by satellite navigators, does not provide outstanding performance; (ii) Occupancy-based criteria tend to be robust and to consistently support a good redistribution of vehicles; and (iii) criteria based on predictions can improve network conditions, particularly when all the vehicles follow an expected behaviour.

We see several avenues for future work. We are interested in evaluating the criteria in different urban regions; however, the structure of Milton Keynes central may suggest that in grid-structure city centres, like American cities, results 
may be very similar. We plan also to consider more computationally expensive rerouting criteria, with the aim of identifying the best ratio between computability and accuracy. Finally, we are starting discussions with UK councils to perform real-world trials.

\section{Acknowledgement}

Mauro Vallati was partially funded by the EPSRC grant EP/R51343X/1 (AI4ME). Lukáš Chrpa was partially funded by the Czech Science Foundation (project no. 18-07252S).

\section{References}

1. Bretherton, R.: Scoot urban traffic control system: Philosophy and evaluation. In: Proceedings of the 6th IFAC/IFIP/IFORS Symposium on Control, Computers, and Communications in Transportation (1989)

2. Chrpa, L., Magazzeni, D., McCabe, K., McCluskey, T.L., Vallati, M.: Automated planning for urban traffic control: Strategic vehicle routing to respect air quality limitations. Intelligenza Artificiale 10, 113-128 (2016)

3. Chrpa, L., Vallati, M., Parkinson, S.: Exploiting automated planning for efficient centralized vehicle routing and mitigating congestion in urban road networks. In: Proceedings of the 34th ACM/SIGAPP Symposium on Applied Computing, SAC (2019)

4. Lopez, P.A., Behrisch, M., Bieker-Walz, L., Erdmann, J., Flötteröd, Y.P., Hilbrich, R., Lücken, L., Rummel, J., Wagner, P., Wießner, E.: Microscopic traffic simulation using sumo. In: 21st International Conference on Intelligent Transportation Systems, ITSC (2018)

5. Lowrie, P.: The sydney coordinated adaptive traffic system-principles, methodology, algorithms. In: Proceedings of the International Conference on Road Traffic Signalling. No. 207 (1982)

6. Manolis, D., Pappa, T., Diakaki, C., Papamichail, I., Papageorgiou, M.: Centralised versus decentralised signal control of large-scale urban road networks in real time: a simulation study. IET Intelligent Transport Systems 12(8), 891-900 (2018)

7. McCluskey, T.L., Vallati, M.: Embedding automated planning within urban traffic management operations. In: Proceedings of the Twenty-Seventh International Conference on Automated Planning and Scheduling (ICAPS). pp. 391-399 (2017)

8. Vallati, M., Chrpa, L.: A principled analysis of the interrelation between vehicular communication and reasoning capabilities of autonomous vehicles. In: 21st International Conference on Intelligent Transportation Systems, ITSC. pp. 3761-3766 (2018)

9. Vallati, M., Magazzeni, D., De Schutter, B., Chrpa, L., McCluskey, T.L.: Efficient macroscopic urban traffic models for reducing congestion: a PDDL+ planning approach. In: The Thirtieth AAAI Conference on Artificial Intelligence (AAAI) (2016)

10. Vincent, R., Pierce, J.: Self-optimising signal control for isolated intersections. In: Crowthorne: Transport and Road Research Laboratory Research Report. No. 170 (1988)

11. Zambrano-Martinez, J.L., Calafate, C.T., Soler, D., Lemus-Zúñiga, L.G., Cano, J.C., Manzoni, P., Gayraud, T.: A centralized route-management solution for autonomous vehicles in urban areas. Electronics 8(7), 722 (2019) 